\title{
Protein Phosphorylation Inhibition
}

National Cancer Institute

\section{Source}

National Cancer Institute. Protein Phosphorylation Inhibition. NCI Thesaurus. Code C41600.

Protein Phosphorylation Inhibition involves interference with, or restraint of, enzymatic activities involved in covalent transfer of a phosphate group from ATP to a protein via the action of a kinase. The major phosphoryl acceptors in proteins are serine, threonine, and tyrosine. Typically, such post-translational modifications regulate protein function. 